\title{
Ekphrasis et figura dans le texte michonien
}

\author{
Sonia Lagerwall (Université de Bergen, Norvège)
}

\begin{abstract}
Pierre Michon's postmodern writing oscillates between the sacred and the simulacrum, the sensible and the intelligible. It prompts the reader's immersion into the text in order to make literature a force that acts. The present article examines this textual specificity in the light of ekphrasis and figura. It argues that Michon revives the rhetorical tradition (ekphrasis) and the Christian tradition (figura) to guide the posture of the reader and establish an aesthetics of performativity, mobilizing an old paradigm (enargeia) where vision is a highly valued sense. The article offers a succinct reading of Abbés (2002).
\end{abstract}

Keywords: Pierre Michon, performativity, ekphrasis, figura, Auerbach

\section{Introduction}

La voix de Pierre Michon est sans conteste une des plus singulières de la littérature contemporaine française. Depuis ses débuts en 1984 avec Vies minuscules cette «énergie de la langue » qualifiée par Jean-Pierre Richard d' «effet-michon» ne cesse de fasciner par sa «vitalité d'énonciation» à haute «teneur émotionnelle» (Richard 2008, 7, 56, 30). Un narrateur «à la fois fraternel et sarcastique » (ibid. 55) aime à adresser le lecteur par des injonctions à voir et à imaginer le monde du texte, suggérant par-là un important potentiel émotif du visuel. Cet article interroge l'ekphrasis et la figura comme des dispositifs qui participent de cette performativité du texte. Ancrées dans la tradition rhétorique et la pensée chrétienne respectivement, l'ekphrasis et la figura sont des formes d'évocation qui disent la volonté de l'écrivain d'inviter le visuel dans le texte pour donner chair à l'écrit. L'œuvre de Michon, qui se résume à une quinzaine de titres publiés à ce jour, atteste d'une même fascination pour la littérature et la peinture. Après un bref rappel de la place qu'occupent les deux arts sœurs dans l'œuvre, le terme ekphrasis sera ici mobilisé dans ses deux acceptions ancienne et moderne - pour le compte de cette écriture. Avec Auerbach, nous évoquerons ensuite la notion de figura qui sera illustrée par une lecture succincte d'Abbés (2002).

\section{Les arts sœurs thématisés}

Dès Vies minuscules, grand «récit de filiation » (Viart 1999, 115-139), la littérature s'impose comme un sujet michonien majeur. Cette «autobiographie oblique et éclatée » (Richard 2008,10) raconte la difficile arrivée en littérature de Michon par le biais de huit récits de vies oubliées dans le village de son enfance en Creuse auxquelles le texte donne des destinées épiques. Michon s'y lance aussi à la recherche du quasi inconnu que fut son père, le grand absent qui avait quitté le foyer en confiant l'enfant aux soins de la mère et des grands-parents. Après les récits de vie de la Creuse, l'autobiographie se poursuit par miroitement à travers une série de portraits d'écrivains admirés avec Rimbaud le fils (1991) - évoquant un autre fils au 
père absent - et Trois auteurs (1997) et Corps du roi (2002) où Michon rend hommage à notamment Faulkner, Beckett, Balzac, Hugo et Flaubert. Par ces portraits d'écrivains, Michon s'inscrit en creux dans une lignée, se taillant une place de fils bâtard dans une filiation littéraire qu'il idéalise et sacralise.

Si le prestige de la littérature engendre le doute et la remise en question permanente chez l'auteur, le rapport à la peinture, en revanche, est plus aisé et riche à la fois de plaisir et de vérité. «La peinture est le grand art d'incarnation» dit-il, c'est le "miracle de la présence réelle » (Michon 2007, 32, 65). Il écrit « environné d'images » et c'est le travail des peintres qu'il consulte le plus souvent quand il faut venir à bout d'un problème d'écriture (ibid. 67, 70) :

« Je voudrais évoquer des hommes avec cet effet presque hallucinatoire qui fait la force des grands portraits. C'est un art d'évocation que je cherche, un art d'apparition. Comme un peintre, c'est une image, une image d'homme, que je veux faire apparaître. » (ibid. 65)

Capitale pour l'écriture, la peinture occupera bientôt une place de prédilection dans l'œuvre. Après Vies minuscules Michon décide d'écrire sur les peintres - « je leur devais bien ça » (ibid. 68). La série sur les écrivains sera ainsi doublée par une série aux vies de peintres tels Van Gogh (Vie de Joseph Roulin, 1988), Goya, Watteau et Piero della Francesca (Maîtres et serviteurs, 1990), et Claude le Lorrain (Le Roi du Bois, 1996). La critique y constate le même phénomène de miroitement autobiographique (cf. Castiglione 2004, 45-57).

\section{L'ekphrasis moderne - la description d'une œuvre d'art visuel}

Dans les textes de Michon sur les peintres on trouve de nombreuses évocations de tableaux célèbres. La représentation verbale d'une œuvre d'art visuel répond de nos jours à la notion d'ekphrasis ; cette définition du terme est cependant moderne et se distingue, comme nous le verrons par la suite, de l'acception ancienne.

Ayant pour sujet l'objet statique d'une œuvre d'art, l'ekphrasis moderne a été théorisée comme un effort de spatialisation dans la linéarité et mobilité du texte. Certains critiques l'ont même pensée comme un instant figé dans le texte, une pause narrative, un arrêt sur image (Krieger, 1992 ; Steiner 1988) ${ }^{1}$. Or chez Michon les tableaux n'ont rien d'immobile, au contraire. A titre d'exemple, La Berceuse, L'Arlésienne et les autres portraits et tableaux de Van Gogh que le lecteur 'voit apparaître' dans Vie de Joseph Roulin génèrent un monde vivant de texte où le peintre, son ami postier et les autres personnages se meuvent par la force de l'imagination de Michon qui les conjure d'être, qui « veu[t] » les voir (Michon 1988, 39).

La plus impressionnante ekphrasis de l'auteur à ce jour reste Les Onze (2009). Michon pousse ici à l'extrême sa conception de la description du tableau. Là où ses ekphraseis antérieures avaient eu pour objet des tableaux réels, il passe ici à la description d'une peinture fictive. Le texte, long de 137 pages, évoque un tableau censé se trouver au Louvre à Paris et représentant les onze membres du Comité de Salut Public par qui la révolution de 1789 tourna à la Terreur. Les Onze est le titre du tableau décrit, ou plutôt narré, car le texte est une longue ekphrasis en forme d'anneau de moebius perfide où les frontières entre représentation et réel sont incertaines et où il existe des trappes qui mènent vers d'autres tableaux, réels cette fois-ci. Un narrateur omniprésent accompagne le lecteur debout devant le tableau au musée du Louvre. Ce topos d'une visite guidée dans une galerie de peintures fait bien sûr écho aux Imagines de

\footnotetext{
${ }^{1}$ Cette conception a été contestée, par exemple par Heffernan (1993) pour qui l'ekphrasis est toujours dynamique et narrative.
} 
Philostrate, texte du troisième siècle de notre ère et une ekphrasis ancienne couramment citée à l'appui de la définition moderne du terme du fait de son sujet pictural. Comme chez Philostrate, le narrateur de Michon incite le lecteur à s'immerger avec lui dans l'image : "Vous imaginez cela, Monsieur ?»; "vous pouvez mettre tout cela à la fois devant les yeux de votre esprit? [...] vous pouvez voir cela ?» (Michon 2009, 20, 21. Nous soulignons).

Souvent sollicitée par les écrivains en métacommentaire sur la littérature, dans un jeu spéculaire où un art permet de réfléchir sur un autre, l'ekphrasis moderne s'est parfois vue associée à une littérature autoréflexive détournée du réel. Si le jeu autoréflexif n'est jamais absent de l'œuvre de Michon, il serait absurde de confiner l'ekphrasis michonienne à la seule fonction métafictionnelle tant l'idée de la peinture comme un accès au réel est primordiale chez lui :

\footnotetext{
« Je suis un homme pour qui le monde visible existe - je dirais même que je ne crois que ce que je vois (et en littérature aussi bien : je ne crois qu'aux auteurs qui me donnent à voir un peu du monde). Alors bien sûr la peinture, sa duplication du monde, sa redondance sur le visible, son infini dialogue avec le visible, sa tractation toujours recommencée avec ce qui est, tout cela est pour moi pain bénit : elle me garde de douter du monde, la peinture, ce que ne fait pas toujours la littérature. » (Michon 2007, 69)
}

Le parti pris du réel est essentiel pour Michon qui se dit peu apte à faire de la fiction pure et d'inventer des personnages de toutes pièces ; l'attache au réel, à l'histoire concrète lui est nécessaire. Il ne s'agit pas pour lui de décrire de façon ressemblante, mimétique, mais d'évoquer un monde dans une langue capable d'émouvoir comme le peut la peinture.

Mobiles et vivaces, émouvantes et insistantes dans leur adresse au lecteur, les ekphraseis de Michon ont beaucoup de traits en commun avec l'acception ancienne et première du terme qui est rappelée par l'ouvrage de Ruth Webb (2009) sur l'ekphrasis rhétorique aux premiers cinq siècles de notre ère.

\section{L'ekphrasis rhétorique - un art d'évocation}

Chez les Anciens le mot ekphrasis ne s'employait guère comme aujourd'hui pour désigner la description verbale d'une œuvre d'art visuel, mais dans un sens beaucoup plus large pour désigner un 'art de parler' créant un effet de vivacité tel que les auditeurs avaient l'impression de se trouver en présence de la scène décrite. Analysant des textes de rhétorique clé comme les Progymnasmata, manuels d'exercices oratoires grecs, et 1'Institutio oratoria de Quintilien, Webb montre la place fondamentale qu'occupait l'ekphrasis dans l'art oratoire. L'ekphrasis était un exercice élémentaire des Progymnasmata visant à transformer l'auditeur en spectateur, à mettre sous les yeux de son esprit la chose narrée par une description vive. Les sujets susceptibles d'être traités dans une ekphrasis englobaient toutes sortes de phénomènes : des personnes, lieux, époques et évènements, et si des objets d'art pouvaient y figurer, ils ne faisaient jamais partie de la liste des sujets généraux.

«[Ekphrasis] is a form of vivid evocation that may have as its subject-matter anything - an action, a person, a place, a battle, even a crocodile. What distinguishes ekphrasis is its quality of vividness, enargeia, its impact on the mind's eye of the listener who must, in Theon's words, be almost made to see the subject» (Webb, 1999, 13). 
Au cœur de l'ekphrasis rhétorique se trouve la notion d'enargeia, vivacité. C'était en imprimant une image mentale dans les esprits des auditeurs que l'orateur pouvait émouvoir son public et lui donner l'impression de participer à la scène. Il s'agit donc d'une pratique ancrée dans un paradigme esthétique qui tenait la vision pour le sens le plus noble et qui valorisait la sphère du visible comme primordiale pour la fonction cognitive et émotive (Webb 2009, 536, 5962, 6001).

Pour les Anciens, l'ekphrasis impliquait le mouvement, une fonction narrative très forte ancrée dans le visuel. Les quatre versions des Progymnasmata insistent toutes dans leurs définitions de l'exercice sur la dimension de dynamique narrative et de parcours qui est la marque de l'ekphrasis rhétorique :

«Ekphrasis is a descriptive (periēgēmatikos) speech which brings (literally 'leads') the thing shown vividly (enargōs) before the eyes» (Les Progymnasmata de Théon, cité et traduit in Webb, 2009, 1256).

Webb souligne ici l'écart qu'il y a avec notre époque dans la conception du descriptif des Anciens qui ne pensaient la description ni comme réservée aux objets, ni comme statique :

«The adjective periēgēmatikos [...] casts the speaker as a guide showing the listener around the sight to be described » (ibid. 1327). ${ }^{2}$ Elle poursuit : « Periēgēsis would therefore suggest a more elaborate form of telling, a winding path instead of the direct through-route of diēgēsis» (ibid. 1333).

Pareillement intéressant pour le compte de Michon est le rôle attribué à l'orateur dans les manuels :

«They also insist on the agency of the speaker as performer or producer, whether he is leading his audience on a virtual tour or conjuring up the subject matter on a virtual stage» (ibid. 1347).

On le voit : avec sa composante d'enargeia, l'ekphrasis rhétorique est loin d'enfermer le visuel dans l'immobilité et le statique - comme aime parfois le faire l'ekphrasis moderne quand est souligné un effet de pause narrative.

Comment cette définition large et inclusive de l'ekphrasis a-t-elle pu être supplantée par l'acception restreinte actuelle du terme ? Webb explique le passage de l'ekphrasis ancienne à l'ekphrasis moderne par un changement de climat intellectuel et esthétique, sensible notamment à partir du XIXe siècle quand écrivains et critiques témoignent d'un intérêt grandissant pour des préoccupations interartielles et pour la comparaison entre les arts amorcée à la Renaissance sous le nom de Paragone. ${ }^{3}$ Webb date «l'invention » de l'ekphrasis moderne à 1955, à un moment où l'influence du structuralisme et de la Nouvelle Critique [New Criticism] imposent une conception du texte comme artefact, éloignant la critique moderne de la conception de la langue comme une force agissant sur l'auditeur qui avait informé la rhétorique gréco-romaine au début de notre ère. ${ }^{4}$ Le romaniste Leo Spitzer est celui par qui le sens moderne restreint « s'invente », explique Webb (752): avec sa célèbre lecture du poème de Keats «Ode on a

\footnotetext{
${ }^{2}$ Aussi les Imagines de Philostrate vont-elles troubler les commentateurs du XIXe siècle qui n'y verront pas une véritable réussite descriptive, tant l'attendu 'arrêt sur image' leur paraîtra absent (Webb 1999, 14).

${ }^{3}$ Pour un historique plus ample de l'invention du genre moderne ekphrasis, voir Webb, 1999.

${ }^{4}$ « The discussions of ekphrasis [...] reveal the strength of the conception of language as a power acting upon the world that was current throughout antiquity. » (Webb, 2009, 627-630)
} 
Grecian Urn » Spitzer redéfinit l'ekphrasis et opère le glissement radical d'une acception basée non plus sur l'effet produit mais sur le sujet décrit : «ekphrasis, [is] the poetic description of a pictorial or sculptural work of art » (Spitzer cité in Webb 2009, 741).

Quand bien même les définitions moderne et ancienne de l'ekphrasis divergeraient sur des points fondamentaux, Webb souligne qu'elles sont généalogiquement liées : dans toutes deux prime le visuel. Dans l'une, toutefois, ce visuel est à chercher dans le référent du texte, alors que dans l'autre il concerne l'effet produit sur l'auditeur/le lecteur :

«T] The different role of the visual is key to the profound differences between the
conceptions underlying the two definitions. For the modern definition the visual is a quality
of the referent, which in some definitions is already a representation of reality. For the
ancient rhetoricians the impact of ekphrasis is visual; it is a translation of the perceptible
which mimics the effect of perception, making the listener seem to see» (Webb 2009, 809).

Depuis l'article révolutionnaire de Spitzer son acception restreinte du terme ekphrasis s'est imposée comme celle ayant cours dans le domaine de l'esthétique. Qu'en est-il advenu de l'acception première ? Les manuels de rhétorique modernes réservent notamment à la figure nommée hypotypose les caractéristiques de l'enargeia consistant à mettre sous les yeux du spectateur la scène décrite. ${ }^{5}$ Aussi est-ce à cette figure que l'art d'évocation de Michon a couramment été identifié par la critique michonienne (cf. Goux 2004, 169-174). Dans l'introduction du numéro des Cahiers de l'Herne récemment consacré à l'auteur, Agnès Castiglione pointe de nouveau la figure :

«Pierre Michon recherche dans le langage le miracle de la présence réelle et pratique cet art d'évocation qui postule la réincarnation par le Verbe. Son projet littéraire est de faire surgir ses personnages du néant par le travail d'une langue capable de susciter les formes par la puissance de l'enargeia, ou « vive représentation », dont la figure de prédilection est l'hypotypose, prouesse « visuelle » plaçant l'image sous les yeux » (Castiglione, 2017, 14).

Aussi pertinente que soit la figure de l'hypotypose pour désigner des scènes de présence réelle et d'incarnation dans l'œuvre de Michon, il nous semble que l'ekphrasis rhétorique révélée par Ruth Webb a de quoi apporter une lumière essentielle sur cette écriture. Pierre Michon partage avec l'esthétique des Anciens une même conception de la vision comme un sens primordial pour la cognition et l'émotion. Cette conception explique sans doute le recours constant au sujet de la peinture dans son œuvre et l'abondance d'ekphraseis au sens moderne du terme : « [L]a peinture [...] n'est pas pour moi, ou pas seulement, plaisir d'esthète, ni objet de savoir - mais inducteur de connaissance, vérité révélée » (Michon 2007, 68-69). L'intérêt qu'il y a ici à rappeler l'ancienne acception de l'ekphrasis est donc double : d'une part, elle permet de mettre l'accent sur l'importance du visuel chez Michon et sur la vision comme un sens actif, dynamique et opérateur d'émotion ; d'autre part, elle a aussi de quoi éclairer son usage idiosyncratique de l'ekphrasis dans le sens moderne du terme où le refus de l'image statique touche à son apogée dans Les Onze, un texte-image infiniment en mouvement. Ce refus du statique dans la description est partiellement l'héritage du Nouveau Roman des années 1950

\footnotetext{
${ }^{5}$ «Hypotypose est un mot grec qui signifie image, tableau. C'est lorsque dans les descriptions on peint les faits dont on parle, come si ce qu'on dit étoit actuèlement devant les yeux ; on montre, pour ainsi dire, ce qu'on ne fait que raconter; on done en quelque sorte l'original pour la copie, les objets pour les tableaux : vous en trouverez un bel exemple dans le récit de la mort d'Hippolyte [dans Phèdre de Racine] » (Dumarsais-Fontanier, 1967 [1818], 151).
} 
et 1960 en France, mais tout porte à croire que Michon puise aussi à ce sujet chez les Anciens pour lesquels il ne cache pas son admiration (ibid. 73, 312).

\section{La figura - le monde dans l'optique chrétienne}

L'ekphrasis rhétorique partage avec notre deuxième notion clé, la figura, l'idée du langage comme une force qui agit sur le monde. La figura, si elle est sémantiquement liée à la figure $^{6}$, ne peut pourtant se confondre avec celle-ci tant elle est ancrée dans la pensée chrétienne. Dans son essai séminal Figura (1938), Erich Auerbach retrace l'évolution du terme latin depuis son acception première de « forme plastique » chez Térence et des auteurs du $\mathrm{I}^{\mathrm{er}}$ siècle av. J.-C., à son apparition comme «prophétie en acte » chez les Pères de l'Église, en passant par la figure de discours des rhétoriciens. La figura, dans le sens que nous souhaitons ici actualiser pour le compte de Michon, appartient donc à l'héritage intellectuel et esthétique du christianisme. Michon dit avoir découvert le texte de la Bible tardivement, à l'âge de 18 ans (Michon 2007, 311). Depuis, il ne cesse de l'interroger et de le relire. " [J]'étais et suis toujours un athée mal convaincu » prétend le narrateur des Vies minuscules (Michon 1984, 204), alors que l'auteur avoue sans détour que : « La Bible est, bien davantage que les Grecs, ma formation, mon imaginaire, mon pays » (Michon 2007, 312). L'on note avec intérêt comment Michon justifie, dans l'entretien cité, sa prédilection pour la pensée chrétienne par rapport à la grecque : après avoir rappelé son admiration pour les historiens et tragédiens grecs, il convoque l'analyse des deux cultures que propose Auerbach au début de Mimésis et souligne la différence en termes d'horizontalité versus verticalité : «[D]ans Homère, tout est sur le même plan ; du détail microscopique à la fresque, c'est un récit frontal qui ne me m'épargne rien, [...] une narration horizontale où tout se vaut. La Bible est verticale » (ibid. 313). Il s'explique : «L'ordre, le calme, les relations apaisées entre le Cosmos et le sujet [comme on en trouve chez les Grecs], je n'y crois pas « (ibid. 314).

Un autre aspect clé qui fait de la Bible un texte de premier plan pour l'écrivain est l'adresse directe au lecteur :

«L'essentiel est dans la dramaturgie biblique à deux voix, Yahvé et son peuple. Ce qui me plaît, c'est que je suis cet interlocuteur auquel s'adresse le texte. [...] Le texte d'Homère est très beau, indiscutablement, mais par le fait qu'il se suffit à lui-même, il est mort [...]. Tout à l'inverse, le texte de la Bible est à vif, il ne se suffit pas. Il est en manque de moi. C'est une affaire d'énonciation. Et cela est au cœur de ce qui me précipite quelquefois à écrire » (Michon 2007, 314-315).

Dans la partie de cet article consacrée à l'ekphrasis nous avons vu la pertinence pour l'écriture de Michon d'une notion comme enargeia (vivacité). Les termes par lesquels il désigne l'énonciation de la Bible y font clairement écho : une « incandescence [...] perfore le texte biblique »; «C'est de l'énergie à l'état pur »; « la vitalité guerrière de la Bible [...] ce tropplein d'énergie et [...] cette formidable sommation que nous assène le texte biblique » (ibid. $314,316)$. Nous souhaitons donc pour finir actualiser la figura comme une seconde dimension importante qui puisse éclairer la voix singulière de Michon et la performativité du texte.

C'est avec la théologie chrétienne dans le cadre du dogme de l'Incarnation, le Verbe qui se fait chair, que s'instaure une acception de la figura qui va dominer la culture occidentale et

\footnotetext{
${ }^{6}$ L'importance de la notion de figure dans l'œuvre de Michon a maintes fois été soulignée dans des lectures riches et variées, en particulier par Dominique Viart (2003 et 2013).
} 
fournir une structure de pensée et d'interprétation du réel et de l'histoire qui prévaudra du Moyen Âge jusqu'au XVIIIe siècle (Auerbach 1993 [1944], 70). C'est par les pères de l'église que s'établit cet emploi de la figura comme une «prophétie en acte » (ibid. 31-54) permettant une lecture du Nouveau Testament qui ne réduit pas l'Ancien Testament à la seule allégorie, mais lui laisse et son historicité et sa signification profonde. Par le principe de la préfiguration un événement dans le premier sera interprété comme la figura (la forme) qui annonce sa réalisation dans le second. Ainsi, Adam ou Moïse dans l'Ancien testament deviennent des figurae du Christ dans le Nouveau, tout comme l'arche de Noé préfigure l'Église (ibid. 42). Figure et accomplissement ont chacun leur réalité historique propre, donc leur individualité, tout en restant interdépendants et reliés par une même essence (ibid. 54) :

\footnotetext{
«La figura est quelque chose de réel et d'historique qui représente et annonce autre chose de tout aussi réel et historique. C'est une concordance ou une ressemblance qui permet de discerner la relation entre les deux événements » (ibid. 32-33).

«L'interprétation figurative établit un rapport entre deux événements ou deux personnes. Le premier terme n'est pas seulement autoréférentiel, mais désigne également le second qui, de son côté, inclut ou accomplit le premier» (ibid. 60).
}

Les deux pôles sont des aspects variables d'une essence impérissable. La figure et son accomplissement sont à la fois même et autre, répétition et différence. De l'une à l'autre il y a, à la fois, statique et dynamique, tradition et nouveauté, codage et création. C'est une structure cyclique de répétition et d'identité qui rappelle le mode opératoire dans beaucoup de textes michoniens. Mais la structure s'affiche peut-être avec le plus d'évidence dans Abbés (2002), un recueil de trois textes sur le monde médiéval chrétien avec ses débats autour de l'Incarnation et le Verbe devenu chair. Dans Abbés les éléments se répondent et s'informent selon le principe de la figura. Il s'agit d'une structure réflexive privilégiant la construction typologique qui, tout en gardant l'abstraction de l'écriture, incite le lecteur à interpréter le texte en fonction des ressemblances entre formes, comme on lit une image.

\section{Abbés}

«Toute la pensée analogique, qui s'étend à tous les domaines de l'esprit médiéval, est étroitement liée à la structure du figurisme » (Auerbach 1993 [1944], 71)

Abbés contient trois brefs récits de vie qui s'inspirent de chroniques médiévales situées en Vendée sur la côte atlantique. Le pays ici est marécageux, eau et terre se rencontrent et «toutes choses sont muables et proches de l'incertain » comme le souligne le refrain qui parcourt les trois histoires (Michon 2002, 32 ; 52 ;71). Un narrateur qui partage avec Michon des traits saillants interroge de vieilles chroniques pour nous faire remonter au Moyen Âge au temps du rayonnement de l'ordre de Cluny vers l'an mil. L'incipit attire d'emblée l'attention sur l'importance de ce double temps qui informe le récit: «JE TIENS de chroniques de seconde main, de la Statistique générale de la Vendée imprimée à Fontenay-le-Comte en 1844, et d'un hasard tardif de ma propre vie, le récit que je m'apprête à raconter» (ibid. 9).

Plantées dans un même décor géographique, ces trois histoires sur autant de générations d'hommes s'articulent entre elles de sorte à inviter à une lecture transversale. Une série de motifs et thèmes sera légèrement altérée d'une histoire à l'autre, alors que le refrain reste 
constant et des mêmes noms de personnages semblent faire office de chainons entre les récits. Bien sûr, dans ce pays boueux où les choses sont muables, rien n'est moins certain que la corrélation entre un nom et l'individu qui le porte : les trois Hugues des trois récits ne renvoient pas à une même personne, alors que les deux Théodolin du livre sont de toute évidence une version jeune et âgée du même homme. Quant aux Pierre, nous y reviendrons. Au moyen de métalepses, le narrateur ramène régulièrement son lecteur au temps présent et incite à lire le passé et le présent en parallèle.

Abbés s'ouvre sur l'histoire d'Èble, frère du belliqueux Guillaume III de Poitiers, dit Guillaume Tête d'étoupe, dont il est le double pacifique. Cet abbé bénédictin est en charge de l'abbaye Saint-Michel-en-l'Herme qui s'érige sur une île isolée dans la boue poitevine. L'Abbé Èble est un homme rongé par «deux passions qui viennent du feu : [...] la gloire et la chair» (ibid. 18). La première le conduira à assécher le marais poitevin. Un jour, devant les frères de l'abbaye, Èble demande à Hugues, jeune moine à la voix vibrante, de lire le passage biblique du Troisième Jour de la Création et déclare : «Nous en sommes au deuxième jour. La terre et les eaux ne sont pas démêlées. Le Tohu et le Bohu qui sont là-dessous, nous allons en faire quelque chose sur quoi on peut mettre le pied » (ibid. 15). A l'image de Dieu appelant le sec au Troisième jour, Èble ordonne d'assécher le marais poitevin. Le mode de la figura s'active, pour dire la vanité - ou est-ce la grandeur - de l'homme.

Dans la bibliothèque de l'abbaye, Èble consulte les « livres qui parlent de terre et d'eau » et qui pourraient le guider dans son exploit : «César, Constance ; ceux des historiens [...], Dion Cassius, Tacite ; ceux des touche-à-tout et des agronomes, Pline, [...] et Augustin, qui démontre que la matière et le miracle s'imbriquent comme mortaise et tenon" (ibid. 19. Nous soulignons).

Ce fut Augustin, nous rappelle Auerbach, par qui la figura s'imposa définitivement comme interprétation du monde sensible et historique des hommes dans l'Antiquité tardive et au Moyen Âge. Par son refus devant toute lecture symboliste de l'Ancient Testament, pouvant réduire le réalisme historique du texte, Augustin encouragea l'articulation entre l'histoire et le spirituel qui est constitutive de l'interprétation figurative (Auerbach 1993, 41-54). C'était bien démontrer l'imbrication nécessaire de « la matière et le miracle », comme l'exprime ici Michon.

La deuxième passion d'Èble est celle pour la chair. C'est ici que le texte va explicitement proposer la grille d'interprétation figurative comme modèle de lecture. L'amour de la chair pousse Èble à jouir chaque jour de la belle femme d'un pêcheur venu d'une des îles d'alentours travailler comme main-d'œuvre dans le projet fou de l'abbé. Mais Hugues, le jeune moine à la voix vibrante, est son rival dans la cabane de la femme du pêcheur. Au sortant d'une conversation avec Hugues qui vient de lui confesser ses visites, Èble, rongé par la jalousie, « pense au roi David, à Bethsabée, à Urie le hittite » (Michon 2002, 25). La rivalité entre Èble et Hugues est dès lors mise sous le signe de la jalousie biblique du roi David qui fit tuer son rival Urie, le mari de la belle Bethsabée dont il s'était violemment épris. L'allusion explicite au récit de l'Ancien Testament invite le lecteur à lire les personnages parallèlement comme les accomplissements des figurae bibliques.

Èble assigne Hugues responsable d'une charrue qu'il sait menée par des bœufs troubles. L'accident se produit et le corps de Hugues est coupé en deux. Le motif du corps coupé en deux, réitéré dans chacune des trois histoires d'Abbés, participe des nombreuses occurrences textuelles à travers lesquelles Michon mobilise le principe dual qui est au cœur de sa poétique (Richard 2008, 27, 40), un principe qui informe bien sûr également la figura. 
Hugues a beau être mort, son nom ne cessera de réapparaître dans les histoires suivantes. Idem pour le motif de sa voix vibrante qui se balade librement à travers le livre pour échouer avec fracas dans le dernier récit. Ici le crane de Jean-Baptiste, «le Précurseur, le Supplanté » (Michon 2002, 64) - encore un corps coupé en deux - joue un rôle de premier plan comme une relique au pouvoir puissant. La langue comme force agissant sur le réel - une thématique de choix dans toute l'œuvre de Michon - retrouve avec Jean-Baptiste son contexte théologique.

La dynamique d'une même essence réapparaissant sous diverses formes et identités informe le livre dans son ensemble. Les passions d'Èble pour la gloire et la chair se réitèrent dans le deuxième récit à travers le personnage d'Emma, épouse de Guillaume, fils de Guillaume Tête d'Étoupe. Par souci de gloire elle construira le monastère Saint-Pierre, la deuxième abbaye du golfe qui fera face à Saint-Michel où Èble avait été abbé. Comme lui, elle interprète les signes du monde pour y trouver du sens, et comme lui, elle fera violemment éliminer sa rivale en amour, cette fois-ci pour se retrouver elle-même dans l'eau comme un corps coupé en deux.

L'incipit et les métalepses du narrateur rappellent régulièrement le double temps qui agit dans Abbés. Ainsi la structure figurative ne permet-elle pas seulement l'enchevêtrement des trois histoires mais aussi à «un fort mouvement de sympathie, de compassion, d'équivalence » de s'établir entre le Moyen Âge et le présent, pour paraphraser Michon questionné sur le dialogue avec l'Autre dans son autobiographie oblique (Michon 2007, 67). La passion d'Èble pour la chair se solde, des années plus tard, par une enfant blonde à tête d'étoupe que l'abbé reconnaît comme la sienne dans la barque qui transporte la famille du pêcheur venue fournir l'abbaye en poissons pour la table de l'Épiphanie. Comme Pierre Michon lui-même, Èble sera donc doté d'un enfant sur le tard, une fille qui, à l'heure de la mort de l'abbé, lui apparaît comme la chair qui incarne cette « gloire » recherchée dans l'asséchement du marécage (Michon 2002, 32). Dans le texte intitulé «Le ciel est un très grand homme » (Corps du roi), Michon avait convoqué la figura du Booz biblique à travers le poème de Victor Hugo «Booz endormi » pour dire la naissance miraculeuse de sa propre fille.

Mais l'interprétation figurative rapprochant Michon à ses personnages dans Abbés ne s'arrête pas à la glorieuse paternité tardive que l'auteur a en commun avec Èble. Le deuxième récit commence ainsi :

«Je tiens de Pierre de Maillezais - qui sûrement ne s'appelait pas Pierre mais avait choisi
ce prénom monastique en renonçant au monde, et qui n'était pas davantage de Maillezais
[...]-et qui écrivit sa Chronique de Maillezais dans les années où Guillaume, petit-fils de
Guillaume Longue-épée, depuis le chenil d'Hastings déchaînait ses meutes sur
l'Angleterre, de cet hybride donc, ou de cette forgerie, de ce pur nom, je tiens le récit que
je vais dire» (Michon 2002, 35. Nous soulignons.)

Le nom à la fois « hybride » et « pur » de Pierre, que l'auteur/narrateur partage avec le chroniqueur médiéval sur lequel il s'appuie pour l'autorité de son récit, est la « forgerie » du texte. Agnès Castiglione (2004, 45-57) a mis la lumière sur ce jeu de miroir entre l'auteur et ses nombreux personnages appelés Pierre, souvent calqués sur des modèles historiques, qui traverse l'œuvre depuis Vies minuscules. Le principe de l'autobiographie oblique qui consiste à «passer par l'autre » «être l'autre dans un fort mouvement de sympathie, de compassion, d'équivalence » pour "se mettre soi-même en lumière, en souffrance, en épreuve » (Michon 2007, 67) est donc à l'œuvre également dans Abbés, un texte qui promeut en principe épistémologique le mouvement réflexif et évolutif de la figura où une même essence se 
maintient à travers des formes différentes. Le lecteur de Michon s'immerge dans le texte comme dans une image, construisant le sens au moyen des ressemblances et différences de formes.

Nous avons ici tenté de penser la performativité du texte michonien à partir de la double perspective ekphrastique (rhétorique) et figurative (chrétienne). L'analyse a montré que Michon partage avec la rhétorique ancienne et la pensée chrétienne une conception de la langue comme une force agissant sur le réel où l'image et des procédés visuels sont fortement valorisés pour une réception cognitive et émotive. Nous avons vu que l'usage que fait Michon des modes anciens s'inscrit dans une littérature post-Nouveau-Roman qui est des plus contemporaines. Aussi réinvente-t-il de façon critique les dispositifs textuels que sont l'ekphrasis et la figura : dans l'une comme dans l'autre le lecteur est cet interlocuteur dont le texte est en manque.

\section{Bibliographie}

Auerbach. Erich. 1993 [1944]. Figura. Traduit par Marc André Bernier. Paris : Éditions Belin. Castiglione, Agnès. 2004. «"Tu connais Pierrot": un autoportrait de 1'artiste ». In Pierre Michon, l'écriture absolue, Agnès Castiglione (éd.), 45-57. Saint-Étienne : Publications de l'Université de Saint-Étienne.

---, 2017. « Avant-propos ». In Pierre Michon, Agnès Castiglione, Dominique Viart et Philippe Artières (éds.), 13-15. Paris : Éditions de L'Herne.

Dumarsais-Fontanier. 1967 [1818]. Les Tropes tome 1. Genève : Slatkine Reprints.

Goux, Jean-Paul. 2004. «La scène fantasmée dans Rimbaud le fils». In Pierre Michon, l'écriture absolue, Agnès Castiglione (éd.), 169-174. Saint-Étienne : Publications de l'Université de Saint-Étienne.

Heffernan, James. 1993. Museum of Words: The Poetics of Ekphrasis from Homer to Ashbery. Chicago : University of Chicago Press.

Krieger, Murray. 1992. Ekphrasis: The Illusion of the Natural Sign. Baltimore: The John Hopkins University Press.

Michon, Pierre. 1984. Vies minuscules. Paris : Gallimard.

---, 1988. Vie de Joseph Roulin. Lagrasse : Éditions Verdier

---, 1990. Maîtres et serviteurs. Lagrasse : Éditions Verdier.

---, 1991. Rimbaud le fils. Paris : Gallimard.

---, 1996. Le Roi du Bois. Lagrasse : Éditions Verdier.

---, 1997. Trois auteurs. Lagrasse : Éditions Verdier.

---, 2002. Corps du roi. Lagrasse : Éditions Verdier.

---, 2007. Le Roi vient quand il veut. Propos sur la littérature. Paris : Albin Michel.

---, 2009. Les Onze. Lagrasse : Éditions Verdier.

Richard, Jean-Pierre. 2008. Chemins de Michon. Lagrasse : Éditions Verdier.

Spitzer, Leo. 1955. "The "Ode on a Grecian Urn," or content vs. Metagrammar." Comparative Literature 7: 203-25.

Steiner, Wendy. 1988. Pictures of Romance : Form against Context in Painting and Literature. Chicago : University of Chicago Press. 
Viart, Dominique. 1999. «Filiations littéraires ». In Écritures contemporaines 2. État du roman contemporain, Jan Baetens et Dominique Viart (éds.), 115-139. Paris-Caen : Minard.

---, 2003. «Pierre Michon : un art de la figure ». In Pierre Michon entre pinacothèque et bibliothèque, Ivan Farron et Karl Kürtös (éds.), 15-33. Berne : Peter Lang.

---, 2013. «Poétique de la figuration dans l'œuvre de Pierre Michon ». In Pierre Michon. La lettre et son ombre, Pierre-Marc de Biasi, Agnès Castiglione et Dominique Viart (éds.), 783-1263. Paris : Gallimard. Kindle.

Webb, Ruth. 1999. "Ekphrasis ancient and modern : The invention of a genre." Word \& Image 15 (1): 7-18.

---, 2009. Ekphrasis, Imagination and Persuasion in Ancient Rhetorical Theory and Practice. Farnham: Ashgate Publishing Ltd. Kindle. 